\title{
Generalized Net Model for Telecommunication Processes in Telecare Services
}

\author{
MIKHAIL MATVEEV, VELIN ANDONOV, \\ KRASSIMIR ATANASSOV (IEEE Member) \\ Institute of Biophysics and Biomedical Engineering \\ Bulgarian Academy of Sciences, Sofia, BULGARIA
}

\author{
MARIA MILANOVA \\ Multi-profile Hospital for Active Medical Treatment \\ and Emergency Medicine "N.I.Pirogov" \\ Sofia, BULGARIA
}

\begin{abstract}
In a series of papers, Generalized Net (GN) models of processes, related to tracking changes in the health status of adult patients, have been presented. The contemporary state-ofthe- art of the telecommunications and navigation technologies allow these models to be further extended to the case of active and mobile patients. This requires the inclusion of patient's current location as a new and significant variable of the model. Various opportunities are considered for the retrieval of this information, with a specific focus on the optimal ones, and a refined GN model is herewith proposed.
\end{abstract}

Keywords: Generalized nets, Modelling, Telecare, Telecare services.

Received: July 10, 2021. Revised: December 14, 2021. Accepted: December 27, 2021. Published: January 20, 2022.

\section{Introduction}

In a series of papers, Generalized Net (GN, see the Appendix) models of processes, related to tracking the changes in health status of adult patients have been presented (see, e.g., $[1,2])$. They are a continuation of the ideas for description of processes, taking place in hospital units, using the apparatus of GNs (see, e.g., [3-5]). The so constructed nets give the possibility to model the logical conditions for the realization of the processes, to simulate these processes, as well as search ways for their optimization.

In [1], the processes for generation of signals from sensors around adult patients and their transmission through different telecommunication tools to the respective hospital units, have been described. In [2], a GN-model of the telecommunication processes between the adult patients and hospital units, has been discussed. Below, a GN-model of the processes for signal classification and the reaction of the medical staff of the hospital units, is constructed. In the Appendix, short remarks of GNs are given.

\section{The Generalized Net Model}

The GN model (see Fig. 1) consists of.

- $\quad$ six transitions $Z_{1}, Z_{2}, Z_{3}, Z_{4}, Z_{5}$ and $Z_{6}$.

- sixteen places $l_{1}, l_{2}, \ldots, l_{16}$.

- four different types of tokens representing the patients, the dispatchers that monitor the signals from the sensors, the medical doctors who examine the patients and the medical specialists.

The tokens $\pi_{1}, \pi_{2}, \ldots, \pi_{k}$ which represent the patients enter the net in place $l_{4}$ with initial characteristic "patient; name of the patient; current health status".

The tokens $\delta_{1}, \delta_{2}, \ldots, \delta_{l}$ which represent dispatchers enter the net in place $l_{8}$ with initial characteristic: "dispatcher; name of the dispatcher; information about all received signals”.
The tokens $\mu_{1}, \mu_{2}, \ldots, \mu_{m}$ which represent the medical doctors who examine the patients enter the net in place $l_{9}$ with initial characteristic: "medical doctor; name of the medical doctor; specialty".

The tokens $\sigma_{1}, \sigma_{2}, \ldots, \sigma_{m}$ which represent the medical specialists who examine the patients enter the net in place $l_{9}$ with initial characteristic: "medical doctor; name of the medical doctor; specialty”.

The six transitions will be described in details below.

The first transition $Z_{1}$ has the form:

$$
Z_{1}=\left\langle\left\{l_{4}, l_{10}, l_{15}\right\},\left\{l_{1}, l_{2}, l_{3}, l_{4}\right\}, R_{1}\right\rangle,
$$

where

$R_{1}=$\begin{tabular}{c|cccc} 
& $l_{1}$ & $l_{2}$ & $l_{3}$ & $l_{4}$ \\
\hline$l_{4}$ & false & $W_{4,2}$ & $W_{4,3}$ & $W_{4,4}$ \\
$l_{10}$ & true & false & false & false \\
$l_{15}$ & false & false & false & true
\end{tabular}

and the predicates in the index matrix $R_{1}$ have the meanings:

- $W_{4,2}=$ "the sensor detected a change in patient's condition",

- $W_{4,3}=$ "the patient should be transported to hospital"

- $W_{4,4}=\neg W_{4,2}$,

where $\neg P$ is the negation of the predicate $P$.

When the truth-value of the predicate $W_{4,2}$ is true, the token $\pi_{i}$ enters place $l_{2}$ with characteristic "signal of the sensor about the current patient".

When the truth-value of the predicate $W_{4,3}$ is true, the token $\pi_{i}$ enters place $l_{3}$ with characteristic "name of the patient; current status".

In place $l_{4}$, the $\pi$-tokens receive the characteristic "current status of the patient”. 
WSEAS TRANSACTIONS ON COMMUNICATIONS

DOI: $10.37394 / 23204.2022 .21 .5$

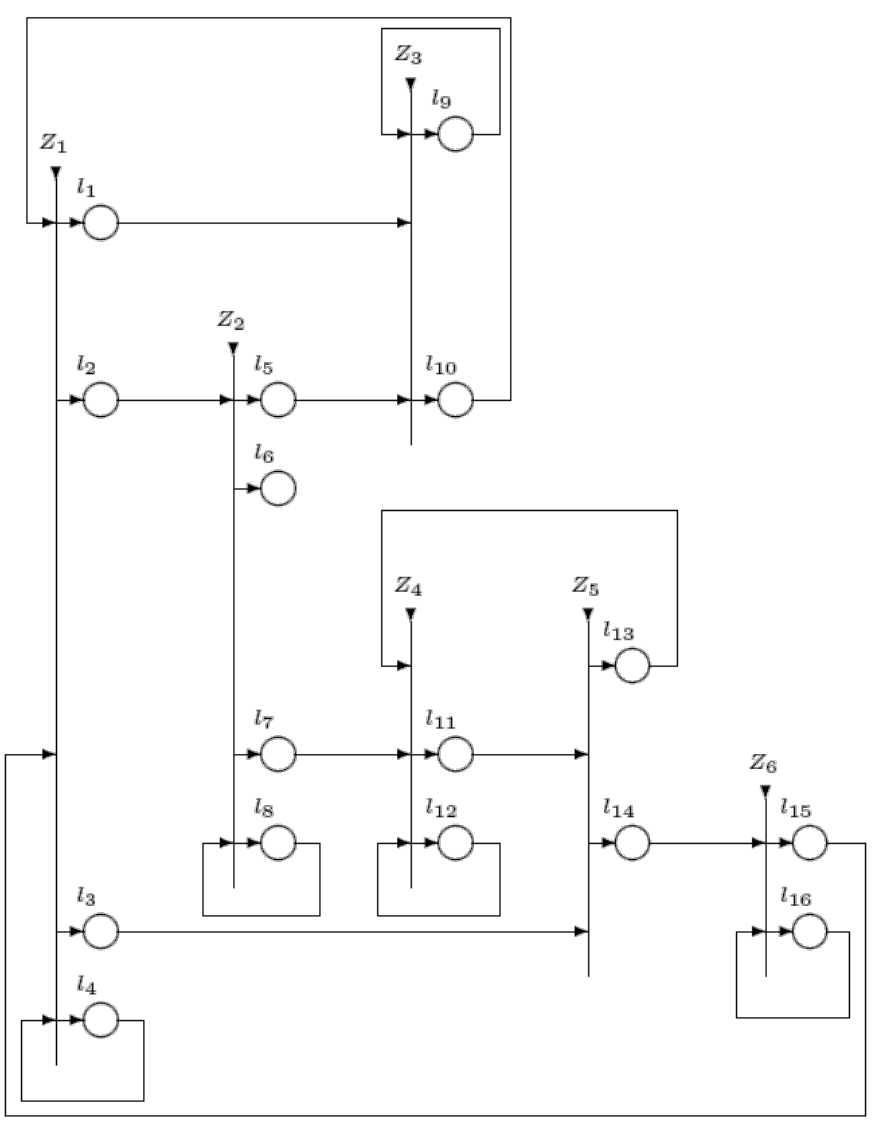

Fig. 1. GN model of the telecommunication processes, taking place in telecare services

The second transition $Z_{2}$ has the following form:

where

$$
Z_{2}=\left\langle\left\{l_{2}, l_{8}\right\},\left\{l_{5}, l_{6}, l_{7}, l_{8}\right\}, R_{2}\right\rangle,
$$

$$
R_{2}=\begin{array}{c|cccc} 
& l_{5} & l_{6} & l_{7} & l_{8} \\
\hline l_{2} & W_{2,5} & W_{2,6} & W_{2,7} & \text { false } \\
l_{8} & \text { false } & \text { false } & \text { false } & \text { true }
\end{array}
$$

and the predicates in the index matrix $R_{2}$ have the meanings:

- $W_{2,5}=$ "medical doctor should be sent to perform examination of the patient at home",

- $W_{2,6}=$ "no action is necessary",

- $W_{2,7}=$ "the patient should be transported to a medical center". When the truth-value of the predicate $W_{2,5}$ is true, the token $\pi_{i}$ enters place $l_{5}$ with characteristic "a decision to visit the patient has been taken".

When the truth-value of the predicate $W_{2,6}$ is true, the token $\pi_{\mathrm{i}}$ enters place $l_{6}$ with characteristic "a decision to ignore the signal has been taken”.

When the truth-value of the predicate $W_{2,7}$ is true, the token $\pi_{i}$ enters place $l_{7}$ with characteristic "a decision to transport the patient to a medical center has been taken”.

The third transition $Z_{3}$ has the form:

where

$$
Z_{3}=\left\langle\left\{l_{1}, l_{5}, l_{9}\right\},\left\{l_{9}, l_{10}\right\}, R_{3}\right\rangle,
$$

$$
R_{3}=\begin{array}{c|cc} 
& l_{9} & l_{10} \\
\hline l_{1} & \text { true } & \text { false } \\
l_{5} & \text { true } & \text { false } \\
l_{9} & W_{9,9} & W_{9,10}
\end{array}
$$

and the predicates in the index matrix $R_{3}$ have the meanings

- $W_{9,10}=$ "a medical doctor should be sent to examine the patient”,

- $W_{9,9}=\neg W_{9,10}$.

In place $l_{9}$, the $\mu$-tokens do not obtain any new characteristics.

When the truth-value of the predicate $W_{9,10}$ is true, the corresponding $\mu_{i}$ token representing the medical doctor enters place $l_{10}$ with characteristic "name of the medical doctor who will visit the patient”.

The forth transition $Z_{4}$ has the following form:

where

$$
Z_{4}=\left\langle\left\{l_{7}, l_{12}, l_{13}\right\},\left\{l_{11}, l_{12}\right\}, R_{4}\right\rangle,
$$

$$
R_{4}=\begin{array}{c|cc} 
& l_{11} & l_{12} \\
\hline l_{7} & \text { false } & \text { true } \\
l_{12} & W_{12,11} & W_{12,12} \\
l_{13} & \text { false } & \text { true }
\end{array}
$$

and the predicates in the index matrix $R_{4}$ have the meanings

- $W_{12,11}=$ "specialists should be sent to bring the patient to the hospital";

- $W_{12,12}=\neg W_{12,11}$.

In place $l_{11}$ the current token $\sigma_{i}$ receives the characteristic "names of the specialists who will bring the patient to the hospital”.

In place $l_{12}$ the tokens receive the characteristic "names of the staff on duty".

The fourth transition $Z_{5}$ has the form:

where

$$
Z_{5}=\left\langle\left\{l_{3}, l_{11}\right\},\left\{l_{13}, l_{14}\right\}, R_{5}\right\rangle,
$$

$$
R_{5}=\begin{array}{c|cc} 
& l_{13} & l_{14} \\
\hline l_{3} & \text { false } & \text { true } \\
l_{11} & \text { true } & \text { false }
\end{array}
$$

In place $l_{13}$, the tokens receive the characteristic "time for completing the transportation of the patient”.

In place $l_{14}$, the tokens receive the characteristic "condition of the patient upon arrival at the hospital”.

The sixth, final, transition $Z_{6}$ has the following form:

where

$$
Z_{6}=\left\langle\left\{l_{14}, l_{16}\right\},\left\{l_{15}, l_{16}\right\}, R_{6}\right\rangle,
$$

$$
R_{6}=\begin{array}{c|cc} 
& l_{15} & l_{16} \\
\hline l_{14} & \text { false } & \text { true } \\
l_{16} & W_{16,15} & W_{16,16}
\end{array},
$$

and the predicates in the index matrix $R_{6}$ have the meanings 
- $W_{16,15}=$ "all medical procedures are completed";

- $W_{16,16}=\neg W_{16,15}$.

In place $l_{15}$, the current $\pi_{i}$ token receives the characteristic "condition of the patient upon discharge from hospital”.

In place $l_{16}$, the current $\pi_{i}$ token receives the characteristic "condition of the patient during the procedures".

Finally, we mention that place $l_{8}$ represents the processes, described by the GN from [3], while places $l_{14}, l_{15}$ and $l_{16}$ correspond to the processes, modeled by the GN from [4]. On the other hand, the present GN model elaborates into further details the basic idea presented in [5].

\section{Conclusions}

The so constructed GN model traces the logical stages of the final part of the process of communication between the sensors connecting mobile adult patients and the staff of the respective hospital unit. The developed model can be used for simulation of the processes of decision making of the appropriate specialists, who must either visit the respective adult patient or to transport him/her to the hospital unit. The model permits simulation of different scenarios e.g. the situation, in which many patients simultaneously require medical assistance.

\section{Acknowledgment}

This work was partly funded by the project FP7-PEOPLE2009-IRSES-247541-MATSIQEL.

\section{References}

[1] Andonov, V., et al. Generalized Net Model for Telecare Services, IEEE Conf. “Intelligent Systems”, Sofia, Bulgaria, 6-8 Sept. 2012, 221-224.

[2] Andonov, V., T. Stojanov, K. Atanassov, P. Kovachev, Generalized Net Model for Telecommunication Processes in Telecare Services, $1^{\text {st }}$ International Conference on Telecommunications and Remote Sensing, Sofia, Bulgaria, 29-30 Aug. 2012, 158-162.

[3] Chakarov, V., et al. Generalized net model for some basic clinical administrative decision making. $1^{\text {st }}$ European Conference on Health Care Modelling and Computation Craiova, Aug. 31 - Sept., 2, 2005, 72-78.

[4] Matveev M., et al. Dynamic model of intensive care unit workflow based on generalized nets. International Electronic Journal "Bioautomation", Vol. 2, 2005, 85-92.

[5] Shannon, A., et al. The generalized net modelling of information healthcare system. Int. Conf. "Automatics and Informatics'06", Sofia, 3-6 Oct. 2006, 119-122.

[6] Atanassov, K., Generalized Nets, World Scientific, Singapore, 1991.

[7] Atanassov, K., On Generalized Nets Theory, "Prof. Marin Drinov" Publishing House of the Bulgarian Academy of Sciences, 2007.

\section{APPENDIX: SHORT REMARKS ON GENERALIZED NETS}

Generalized Nets (GNs, see [6,7] are extensions of the apparatus of mathematical modelling of Petri Nets and other modifications of theirs. GNs are a tool intended for the detailed modelling of parallel and concurrent processes.

A GN is a collection of transitions and places ordered according to some rules (see Fig. 2). The places are marked by circles. The set of places to the left of the vertical line (the transition) are called input places, and those to the right are called output places. For each transition, there is an index matrix with elements called predicates. Some GN-places contain tokens - dynamic elements entering the net with initial characteristics and getting new ones while moving within the net. Tokens proceed from an input to an output place of the transition if the predicate corresponding to this pair of places in the index matrix is evaluated as "true". Every token has its own identifier and collects its own history that could influence the development of the whole process modelled by the GNs.

Two time-moments are specified for the GNs: for the beginning and the end of functioning, respectively.

A GN can have only a part of its components. In this case, it is called reduced $G N$. Here, we shall give the formal definition of a reduced GN without temporal components, place and arc capacities, and token, place and transition priorities.

Formally, every transition in the used below reduced GN is described by a triple: $Z=\left\langle L^{\prime}, L^{\prime \prime}, r\right\rangle$, where:

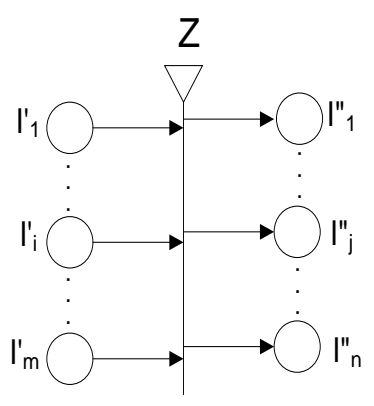

Fig. 2. A GN transition

(a) $\quad L^{\prime}$ and $L^{\prime \prime}$ are finite, non-empty sets of places (the transition's input and output places, respectively); for the transition these are

$$
L^{\prime}=\left\{l_{1}^{\prime}, l_{2}^{\prime}, \ldots, l_{m}^{\prime}\right\} \text { and } L^{\prime \prime}=\left\{l^{\prime \prime}{ }_{1}, l^{\prime \prime}{ }_{2}, \ldots, l_{n}{ }_{n}\right\}
$$

(b) $r$ is the transition's condition determining which tokens will pass (or transfer) from the transition's inputs to its outputs; it has the form of an Index Matrix (IM):

\section{Creative Commons Attribution License 4.0 (Attribution 4.0 International, CC BY 4.0)}

This article is published under the terms of the Creative Commons Attribution License 4.0 https://creativecommons.org/licenses/by/4.0/deed.en_US 


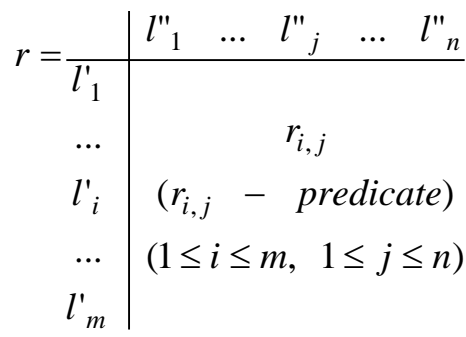

where $r_{i, j}$ is the predicate that corresponds to the $i^{- \text {th }}$ input and $j^{\text {th }}$ output place. When its truth value is "true", a token from the $i^{\text {th }}$ input place transfers to the $j^{\text {th }}$ output place; otherwise, this is not possible.

The ordered four-tuple

$$
E=\langle A, K, X, \Phi\rangle
$$

is called a reduced Generalized Net if:

(a) $A$ is the set of transitions;

(b) $K$ is the set of the GN's tokens;

(c) $X$ is the set of all initial characteristics which the tokens can obtain on entering the net;

(d) $\Phi$ is the characteristic function that assigns new characteristics to every token when it makes the transfer from an input to an output place of a given transition.

Many mathematical operations (e.g., union, intersection and others), relations (e.g., inclusion, coincidence and others) and operators are defined over the GNs. Operators, being of six types (global, local, hierarchical, reducing, extending and dynamic operators) change the structure of the GN, the strategies of token transfer, etc. 